\title{
Exact Solutions of Damped Improved Boussinesq Equations by Extended $\left(G^{\prime} / G\right)$-Expansion Method
}

\author{
Kai Fan (iD ${ }^{1,2,3,4}$ and Cunlong Zhou (iD) ${ }^{1,2,3}$ \\ ${ }^{1}$ Engineering Research Center of Heavy Machinery Ministry of Education, Taiyuan University of Science and Technology, \\ Taiyuan 030024, China \\ ${ }^{2}$ Mechanical Engineering College, Taiyuan University of Science and Technology, Taiyuan 030024, China \\ ${ }^{3}$ Shanxi Provincial Key Laboratory of Metallurgical Device Design Theory and Technology, \\ Taiyuan University of Science and Technology, Taiyuan 030024, China \\ ${ }^{4}$ Applied Science College, Taiyuan University of Science and Technology, Taiyuan 030024, China
}

Correspondence should be addressed to Kai Fan; 2014279@tyust.edu.cn and Cunlong Zhou; zcunlong@tyust.edu.cn

Received 3 June 2020; Revised 1 July 2020; Accepted 8 July 2020; Published 1 August 2020

Guest Editor: Viorel-Puiu Paun

Copyright $(2020$ Kai Fan and Cunlong Zhou. This is an open access article distributed under the Creative Commons Attribution License, which permits unrestricted use, distribution, and reproduction in any medium, provided the original work is properly cited.

\begin{abstract}
With the help of the auxiliary function method, we solved the improved Boussinesq (IBq) equation with fluid dynamic damping and the modified IBq (IMBq) equation with Stokes damping, and we obtained their three types of travelling wave exact solutions, which is an extension service of the numerical simulation and the existence of a solution. From the waveform diagram of IBq equation with hydrodynamic damping, it can be seen that when the propagation velocity of kink wave changes, the amplitude also changes significantly, and it is also found that the kink isolated waveform is significantly asymmetric due to the increase of damping coefficient $v$, which may be of some value in explaining some physical phenomena. In addition, the symbolic computing software maple makes our computing work easier.
\end{abstract}

\section{Introduction}

There are various kinds of nonlinear phenomena in nature, most of which can be described by nonlinear evolution equations. It is well known that the Boussinesq (Bq) equation, which describes the propagation model of long wave in shallow water, is one of them and has the following two basic forms:

$$
\begin{gathered}
u_{t t}-u_{x x}+\delta u_{x x x x}=\left(u^{2}\right)_{x x}, \\
u_{t t}-u_{x x}-u_{x x t t}=\left(u^{2}\right)_{x x},
\end{gathered}
$$

where $u$ represents displacement and subscripts $x$ and $t$ represent partial derivatives concerning $x$ and $t$, respectively. Equations (1) and (2) were first deduced by Boussinesq [1,2]. If the coefficient $\delta$ of the fourth derivative of (1) is greater than zero, (1) is linearly stable and can be used to describe the transverse vibration of small nonlinear elastic beam, which is called the "good" Bq equation [3]. When $\delta$ is less than zero, it is called the "bad" Bq equation because of its linear instability [4]. Equation (2) is also an important model to approximate the propagation of long waves in shallow water, which is called the IBq equation. The exact solution of $\mathrm{Bq}$ equation can be obtained by [5-7]. Makhankov derived the $\mathrm{IBq}$ equation from the fluid dynamics equations of the plasma, which had a modified equation called IMBq equation [8], as shown in (3). In recent years, there have been many papers on the dynamics of solitons in plasma [9-12].

$$
u_{t t}-u_{x x}-u_{x x t t}=\left(u^{3}\right)_{x x} .
$$

Equations (2) and (3) differ only in terms of nonlinear forces. Equation (2) can also be used to describe the kinetic and thermodynamic properties of anharmonic monatomic and diatomic chains [13]. In literature [14], lattice soliton dynamics of a single atomic chain under damping and 
external forces are studied. The IBq equation with hydrodynamic damping term and the IMBq equation with Stokes damping term are obtained, respectively, under the conditions of the third and fourth anharmonic potentials. The IBq equation with hydrodynamical damping term is shown in (3), and the IMBq equation with Stokes damping term is shown in (4). Arévalo et al. [14] obtained the solitary wave solution of (4) with bell-shaped shape through numerical simulation. Naranmandula [15] also obtained an equation similar to (4) when studying the propagation of one-dimensional longitudinal wave in nonlinear microstructural solid and simulated the influence of microstructural effect on the evolution of kinked isolated wave by finite difference method:

$$
\begin{gathered}
u_{t t}-u_{x x}-u_{x x t t}-v u_{x x t}=\left(u^{2}\right)_{x x}, \\
u_{t t}-u_{x x}-u_{x x t t}+v u_{t}=\left(u^{3}\right)_{x x} .
\end{gathered}
$$

In 2012, Wang and Xu studied the global existence of small amplitude solutions of the Cauchy problem of (4) in Sobolev space [16]. In 2013, Wang and Xu studied the global existence of small amplitude solutions of the Cauchy problem of (5) in Sobolev space [17]. In 2015, Chen gives sufficient conditions of the blow-up of the solution of the Cauchy problem of (5) in Sobolev space [18]. In order to better grasp the model represented by (4) and (5), it is necessary to obtain their exact travelling wave solutions. With the development of computer technology, many scholars interested in nonlinear science have studied the exact solutions of nonlinear evolution equations and used different methods to solve different equations, such as the Riccati-Bernoulli sub-ODE method [19], the Exp-function method [20], the modified Exp-function method [21, 22], the Exp $(-\phi)$-expansion method [23], the tanh-coth method [24-26], the homogeneous balance method [27], the improvement of $\left(G^{\prime} / G\right)$-expansion method [28-30], the formal linearization method [31], the first integral method [32], $\left(1 / G^{\prime}\right)$-expansion and modified Kudryashov methods [33], and the $\left(G^{\prime} / G\right)$-expansion method [34-38].

Through searching the whole network, we find that there are many articles on Bq equation and also many articles on the existence of solutions to Cauchy problems of (4) and (5). In this paper, by means of the extended $\left(G^{\prime} / G\right)$-expansion method, some exact travelling wave solutions of (4) and (5) are obtained, and some individual solutions are briefly presented and discussed, especially kink soliton solutions assigned to parameters. In the case of an extended $\left(G^{\prime} / G\right)$ expansion, the integral constants of the equation should not be set directly to zero, which may result in the loss of arbitrary constants in the final expression $[38,39]$. Although there is no uniform solution for all nonlinear partial differential equations, different methods may not yield different solutions [40] because these solutions may only be expressed differently or they may be different particular solutions belonging to the same general solution. In addition, some of the different approaches are equivalent [41, 42].

\section{Summary of the Extended $\left(G^{\prime} / G\right)$ - Expansion Method}

Consider a generalized nonlinear evolution equation

$$
P\left(u, u_{x}, u_{t}, u_{t t}, u_{x t}, u_{x x}, \ldots\right)=0,
$$

where $u$ is an unsolved function, and it has two independent variables, $x$ and $t$.

The steps of the extended $\left(G^{\prime} / G\right)$-expansion method to solve (6) are listed as follows:

(i) Step 1. Under the transformation,

$$
u(x, t)=U(\xi), \xi=x-c t .
$$

We translate (6) to the ordinary differential equation (ODE)

$$
P\left(U,-c U^{\prime}, U^{\prime}, c^{2} U^{\prime \prime},-c U^{\prime \prime}, U^{\prime \prime}, \ldots\right)=0
$$

(i) Step 2. If the form of (8) allows, we can integrate it once but do not set the integral constant to zero, which will help simplify the following calculation. The travelling wave solution of (8) is proposed as follows:

$$
U(\xi)=\sum_{i=0}^{m} a_{i}\left(\frac{G^{\prime}}{G}\right)^{i}+\sum_{i=1}^{m} b_{i}\left(\frac{G^{\prime}}{G}\right)^{-i}
$$

where $a_{i}$ and $b_{i}(i=1,2, \ldots, m)$ are undetermined constants. $G$ is a function of $\xi$. In combination with the form of (9), the highest derivative term and the nonlinear term in (8) are balanced by the homogeneous equilibrium principle, and the value of the positive integer $m$ in (9) can be obtained. The $G$ appearing in (8) is the solution of the second-order differential equation

$$
G^{\prime \prime}+\lambda G^{\prime}+\mu G=0
$$

where $\lambda$ and $\mu$ will be determined later.

(iii) Step 3. Substitute (9) and (7) into (8), use the ordinary differential (10) concerning $\left(G^{\prime} / G\right)$ to combine the same power terms of $\left(G^{\prime} / G\right)$, and then set the coefficients of all powers of $\left(G^{\prime} / G\right)$ to zero; we get a nonlinear algebraic system of equations concerning the unknowns $a_{i}, b_{i}, \lambda, \mu$, and $c$.

(iv) Step 4. Using the computational software Maple programming, we can solve the algebraic equations in step 3. By substituting the obtained results into (9) and using the general solutions of (10) in different situations, multiple exact solutions of different types of (6) can be obtained.

The general solutions of (10) are given as 


$$
\left(\frac{G \prime}{G}\right)= \begin{cases}\frac{\sqrt{\lambda^{2}-4 \mu}}{2}\left(\frac{C_{1} \sinh \left(\left(\sqrt{\lambda^{2}-4 \mu} / 2\right) \xi\right)+C_{2} \cosh \left(\left(\sqrt{\lambda^{2}-4 \mu / 2}\right) \xi\right)}{C_{1} \cosh \left(\left(\sqrt{\lambda^{2}-4 \mu}\right) \xi\right)+C_{2} \sinh \left(\left(\sqrt{\lambda^{2}-4 \mu} / 2\right) \xi\right)}\right)-\frac{\lambda}{2}, & \lambda^{2}-4 \mu>0, \\ \frac{\sqrt{4 \mu-\lambda^{2}}}{2}\left(\frac{-C_{1} \sin \left(\left(\sqrt{4 \mu-\lambda^{2}} / 2\right) \xi\right)+C_{2} \cos \left(\left(\sqrt{4 \mu-\lambda^{2}} / 2\right) \xi\right)}{C_{1} \cos \left(\left(\sqrt{4 \mu-\lambda^{2}} / 2\right) \xi\right)+C_{2} \sin \left(\left(\sqrt{4 \mu-\lambda^{2}} / 2\right) \xi\right)}\right)-\frac{\lambda}{2}, & \lambda^{2}-4 \mu<0, \\ \frac{C_{2}}{C_{1}+C_{2} \xi}-\frac{\lambda}{2}, & \lambda^{2}-4 \mu=0 .\end{cases}
$$

These results can further be written in some more simplified forms depending upon the conditions on the ratio of $C_{1}$ and $C_{2}$ as

$$
\left(\frac{G^{\prime}}{G}\right)=\left\{\begin{array}{l}
\frac{\sqrt{\lambda^{2}-4 \mu}}{2} \tanh \left(\frac{\sqrt{\lambda^{2}-4 \mu}}{2} \xi+\xi_{0}\right)-\frac{\lambda}{2}, \lambda^{2}-4 \mu>0, \tanh \left(\xi_{0}\right)=\frac{C_{2}}{C_{1}},\left|\frac{C_{2}}{C_{1}}\right|<1, \\
\frac{\sqrt{\lambda^{2}-4 \mu}}{2} \operatorname{coth}\left(\frac{\sqrt{\lambda^{2}-4 \mu}}{2} \xi+\xi_{0}\right)-\frac{\lambda}{2}, \lambda^{2}-4 \mu>0, \operatorname{coth}\left(\xi_{0}\right)=\frac{C_{2}}{C_{1}},\left|\frac{C_{2}}{C_{1}}\right|>1, \\
\frac{\sqrt{4 \mu-\lambda^{2}}}{2} \cot \left(\frac{\sqrt{4 \mu-\lambda^{2}}}{2} \xi+\xi_{0}\right)-\frac{\lambda}{2}, \lambda^{2}-4 \mu<0, \cot \left(\xi_{0}\right)=\frac{C_{2}}{C_{1}}, \\
\frac{C_{2}}{C_{1}+C_{2} \xi}-\frac{\lambda}{2}, \lambda^{2}-4 \mu=0 .
\end{array}\right.
$$

\section{Exact Solutions of (3) and (4)}

3.1. The IBq Equation with Hydrodynamical Damping

$$
u_{t t}-u_{x x}-u_{x x t t}-v u_{x x t}=\left(u^{2}\right)_{x x}
$$

where $v$ is the damping constant of internal friction (we call this type of friction hydrodynamical).

We apply (7) to (13), we integrate once concerning $\xi$, we set the integration constant to $M$, and then we can convert (12) to nonlinear ODE:

$$
\left(c^{2}-1\right) U^{\prime}-c^{2} U^{\prime \prime \prime}+c v U^{\prime \prime}-2 U U^{\prime}=M .
$$

At equilibrium, the highest nonlinear term in (14), and the highest derivative term in (14), we get $m$ equal to 2 . Therefore, the exact solution of (14) in the form of (9) can be written as follows:

$$
U(\xi)=a_{0}+a_{1} \frac{G^{\prime}}{G}+a_{1}\left(\frac{G^{\prime}}{G}\right)^{2}+b_{1} \frac{G}{G^{\prime}}+b_{2}\left(\frac{G}{G^{\prime}}\right)^{2} .
$$

Substitute (15) into (14), use the ordinary differential (10) concerning $\left(G^{\prime} / G\right)$ to combine the same power terms of $\left(G^{\prime} / G\right)$, and then let the coefficients be equal to zero; we get a nonlinear algebraic system of equations concerning the unknowns $a_{0}, a_{1}, a_{2}, b_{1}, b_{2}, c$, and $M$ : 


$$
\begin{aligned}
& \left(\frac{G}{G^{\prime}}\right)^{5}:-24 c^{2} \mu^{3} b_{2}-4 \mu b_{2}^{2}=0 \\
& \left(\frac{G}{G^{\prime}}\right)^{4}:-6 c^{2} \mu^{3} b_{1}-54 c^{2} \mu^{2} \lambda b_{2}+6 c \mu^{2} v b_{2}-6 \mu b_{1} b_{2}-4 \lambda b_{2}^{2}=0 \\
& \left(\frac{G}{G^{\prime}}\right)^{3}:-12 c^{2} \mu^{2} \lambda b_{1}-38 c^{2} \mu \lambda^{2} b_{2}-40 c^{2} \mu^{2} b_{2}+2 c \mu^{2} v b_{1}+10 c \mu \nu \lambda b_{2}+2 c^{2} \mu b_{2}-4 \mu a_{0} b_{2}-2 \mu b_{1}^{2}-6 \lambda b_{1} b_{2}-2 \mu b_{2}-4 b_{2}^{2}=0 \\
& \left(\frac{G}{G^{\prime}}\right)^{2}:-7 c^{2} \mu \lambda^{2} b_{1}-8 c^{2} \lambda^{3} b_{2}-8 c^{2} \mu^{2} b_{1}-52 c^{2} \mu \lambda b_{2}+3 c \mu \nu \lambda b_{1} \\
& +4 c v \lambda^{2} b_{2}+c^{2} \mu b_{1}+2 c^{2} \lambda b_{2}+8 c \mu v b_{2}-2 \mu a_{0} b_{1}-2 \mu a_{1} b_{2}-4 \lambda a_{0} b_{2}-2 \lambda b_{1}^{2}-\mu b_{1}-2 \lambda b_{2}-6 b_{1} b_{2}=0, \\
& \frac{G}{G^{\prime}}:-c^{2} \lambda^{3} b_{1}-8 c^{2} \mu \lambda b_{1}-14 c^{2} \lambda^{2} b_{2}+c \nu \lambda^{2} b_{1}-16 c^{2} \mu b_{2}+c^{2} \lambda b_{1} \\
& +2 c \mu v b_{1}+6 c v \lambda b_{2}+2 c^{2} b_{2}-2 \lambda a_{0} b_{1}-2 \lambda a_{1} b_{2}-\lambda b_{1}-4 a_{0} b_{2}-2 b_{1}^{2}-2 b_{2}=0 \\
& \left(\frac{G}{G^{\prime}}\right)^{0}: b_{1} c^{2}-2 a_{0} b_{1}+2 a_{2} b_{1} \mu+c v a_{1} \lambda \mu-c^{2} b_{1} \lambda^{2}-2 c^{2} \mu b_{1}-6 c^{2} \lambda b_{2} \\
& +2 c^{2} a_{1} \mu^{2}-c^{2} \mu a_{1}+2 a_{0} a_{1} \mu+2 c v b_{2}+\mu a_{1}+c^{2} a_{1} \lambda^{2} \mu+6 c^{2} a_{2} \lambda \mu^{2}-2 a_{1} b_{2}+c v b_{1} \lambda+2 c v a_{2} \mu^{2}-b_{1}-M=0 \\
& \frac{G^{\prime}}{G}: 14 c^{2} \mu \lambda^{2} a_{2}+c^{2} \lambda^{3} a_{1}+16 c^{2} \mu^{2} a_{2}+8 c^{2} \mu \lambda a_{1}+6 c \mu \nu \lambda a_{2}+c v \lambda^{2} a_{1}-2 c^{2} \mu a_{2} \\
& -c^{2} \lambda a_{1}+2 c \mu v a_{1}+4 \mu a_{0} a_{2}+2 \mu a_{1}^{2}+2 \lambda a_{0} a_{1}+2 \lambda a_{2} b_{1}+2 \mu a_{2}+\lambda a_{1}=0, \\
& \left(\frac{G^{\prime}}{G}\right)^{2}: 8 c^{2} \lambda^{3} a_{2}+52 c^{2} \mu \lambda a_{2}+7 c^{2} \lambda^{2} a_{1}+4 c v \lambda^{2} a_{2}+8 c^{2} \mu a_{1}-2 c^{2} \lambda a_{2}+8 c \mu v a_{2} \\
& +3 c v \lambda a_{1}-c^{2} a_{1}+6 \mu a_{1} a_{2}+4 \lambda a_{0} a_{2}+2 \lambda a_{1}^{2}+2 \lambda a_{2}+2 a_{0} a_{1}+2 a_{2} b_{1}+a_{1}=0 \\
& \left(\frac{G^{\prime}}{G}\right)^{3}: 38 c^{2} \lambda^{2} a_{2}+40 c^{2} \mu a_{2}+12 c^{2} \lambda a_{1}+10 c v \lambda a_{2}-2 c^{2} a_{2}+2 c v a_{1}+4 \mu a_{2}^{2}+6 \lambda a_{1} a_{2}+4 a_{0} a_{2}+2 a_{1}^{2}+2 a_{2}=0 \\
& \left(\frac{G^{\prime}}{G}\right)^{4}: 54 c^{2} \lambda a_{2}+6 c^{2} a_{1}+6 c v a_{2}+4 \lambda a_{2}^{2}+6 a_{1} a_{2}=0 \\
& \left(\frac{G^{\prime}}{G}\right)^{5}: 24 c^{2} a_{2}+4 a_{2}^{2}=0
\end{aligned}
$$


Using symbolic computation software maple, the solution of this system is obtained as follows:

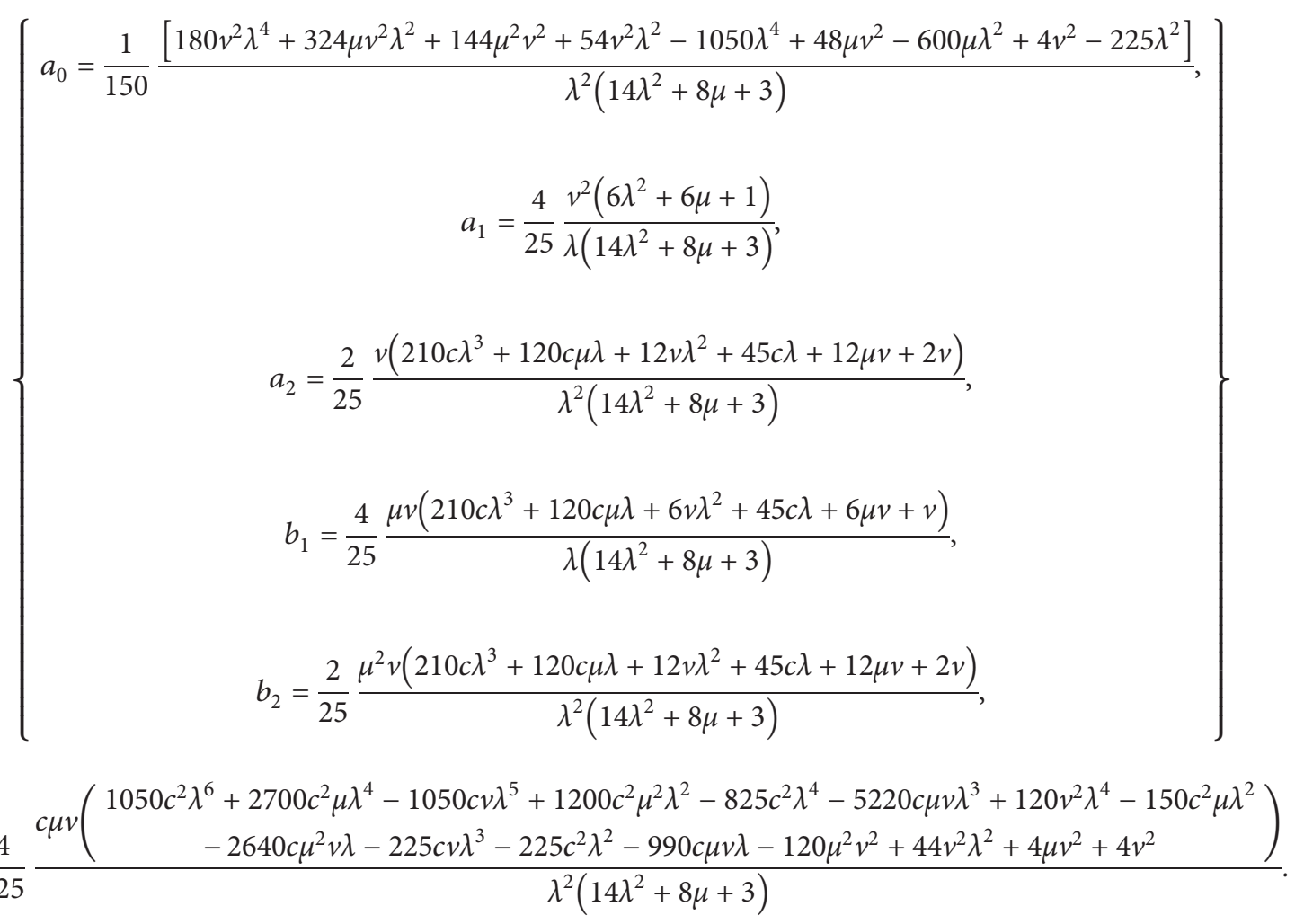

Substituting the values from (17) and using the general solutions of (10) in different situations, multiple exact solutions of different types of (13) can be obtained. (i) Case 1. When $\lambda^{2}-4 \mu>0$, the solution of the hyperbolic form of equation (13) is as follows:

$$
\begin{aligned}
U_{1}(\xi)= & \frac{1}{150} \frac{\left[180 v^{2} \lambda^{4}+324 \mu v^{2} \lambda^{2}+144 \mu^{2} v^{2}+54 v^{2} \lambda^{2}-1050 \lambda^{4}+48 \mu v^{2}-600 \mu \lambda^{2}+4 v^{2}-225 \lambda^{2}\right]}{\lambda^{2}\left(14 \lambda^{2}+8 \mu+3\right)} \\
& +\frac{4}{25} \frac{v^{2}\left(6 \lambda^{2}+6 \mu+1\right)}{\lambda\left(14 \lambda^{2}+8 \mu+3\right)}\left[-\frac{\lambda}{2}+\eta_{1}\left(\frac{C_{1} \sinh \left(\eta_{1} \xi\right)+C_{2} \cosh \left(\eta_{1} \xi\right)}{C_{1} \cosh \left(\eta_{1} \xi\right)+C_{2} \sinh \left(\eta_{1} \xi\right)}\right)\right] \\
& +\frac{2}{25} \frac{v\left(210 c \lambda^{3}+120 c \mu \lambda+12 v \lambda^{2}+45 c \lambda+12 \mu v+2 v\right)}{\lambda^{2}\left(14 \lambda^{2}+8 \mu+3\right)}\left[-\frac{\lambda}{2}+\eta_{1}\left(\frac{C_{1} \sinh \left(\eta_{1} \xi\right)+C_{2} \cosh \left(\eta_{1} \xi\right)}{C_{1} \cosh \left(\eta_{1} \xi\right)+C_{2} \sinh \left(\eta_{1} \xi\right)}\right)\right]^{2} \\
& +\frac{4}{25} \frac{\mu v\left(210 c \lambda^{3}+120 c \mu \lambda+6 v \lambda^{2}+45 c \lambda+6 \mu v+v\right)}{\lambda\left(14 \lambda^{2}+8 \mu+3\right)}\left[-\frac{\lambda}{2}+\eta_{1}\left(\frac{C_{1} \sinh \left(\eta_{1} \xi\right)+C_{2} \cosh \left(\eta_{1} \xi\right)}{C_{1} \cosh \left(\eta_{1} \xi\right)+C_{2} \sinh \left(\eta_{1} \xi\right)}\right)\right]^{-1} \\
& +\frac{2}{25} \frac{\mu^{2} v\left(210 c \lambda^{3}+120 c \mu \lambda+12 v \lambda^{2}+45 c \lambda+12 \mu v+2 v\right)}{\lambda^{2}\left(14 \lambda^{2}+8 \mu+3\right)}\left[-\frac{\lambda}{2}+\eta_{1}\left(\frac{C_{1} \sinh \left(\eta_{1} \xi\right)+C_{2} \cosh \left(\eta_{1} \xi\right)}{C_{1} \cosh \left(\eta_{1} \xi\right)+C_{2} \sinh \left(\eta_{1} \xi\right)}\right)\right]^{-2}
\end{aligned}
$$


where $\xi=x-c t, \eta_{1}=(1 / 2) \sqrt{\lambda^{2}-4 \mu}$, and $C_{1}$ and $C_{2}$ are free constants.
In particular, if $C_{1} \neq 0$ and $C_{2}=0$, then $U_{1}(\xi)$ becomes

$$
\begin{aligned}
\bar{U}_{1}(\xi)= & \frac{1}{150} \frac{\left[180 v^{2} \lambda^{4}+324 \mu v^{2} \lambda^{2}+144 \mu^{2} v^{2}+54 v^{2} \lambda^{2}-1050 \lambda^{4}+48 \mu v^{2}-600 \mu \lambda^{2}+4 v^{2}-225 \lambda^{2}\right]}{\lambda^{2}\left(14 \lambda^{2}+8 \mu+3\right)} \\
& +\frac{4}{25} \frac{v^{2}\left(6 \lambda^{2}+6 \mu+1\right)}{\lambda\left(14 \lambda^{2}+8 \mu+3\right)}\left[-\frac{\lambda}{2}+\eta_{1} \tanh \left(\eta_{1} \xi\right)\right] \\
& +\frac{2}{25} \frac{v\left(210 c \lambda^{3}+120 c \mu \lambda+12 v \lambda^{2}+45 c \lambda+12 \mu v+2 v\right)}{\lambda^{2}\left(14 \lambda^{2}+8 \mu+3\right)}\left[-\frac{\lambda}{2}+\eta_{1} \tanh \left(\eta_{1} \xi\right)\right]^{2} \\
& +\frac{4}{25} \frac{\mu v\left(210 c \lambda^{3}+120 c \mu \lambda+6 v \lambda^{2}+45 c \lambda+6 \mu v+v\right)}{\lambda\left(14 \lambda^{2}+8 \mu+3\right)}\left[-\frac{\lambda}{2}+\eta_{1} \tanh \left(\eta_{1} \xi\right)\right]^{-1} \\
& +\frac{2}{25} \frac{\mu^{2} v\left(210 c \lambda^{3}+120 c \mu \lambda+12 v \lambda^{2}+45 c \lambda+12 \mu v+2 v\right)}{\lambda^{2}\left(14 \lambda^{2}+8 \mu+3\right)}\left[-\frac{\lambda}{2}+\eta_{1} \tanh \left(\eta_{1} \xi\right)\right]^{-2} .
\end{aligned}
$$

Again using (12), the general solutions for $U_{1}(\xi)$ in simplified forms are written as

$$
\begin{aligned}
U_{11}(\xi)= & \frac{1}{150} \frac{\left[180 v^{2} \lambda^{4}+324 \mu v^{2} \lambda^{2}+144 \mu^{2} v^{2}+54 v^{2} \lambda^{2}-1050 \lambda^{4}+48 \mu v^{2}-600 \mu \lambda^{2}+4 v^{2}-225 \lambda^{2}\right]}{\lambda^{2}\left(14 \lambda^{2}+8 \mu+3\right)} \\
& +\frac{4}{25} \frac{v^{2}\left(6 \lambda^{2}+6 \mu+1\right)}{\lambda\left(14 \lambda^{2}+8 \mu+3\right)}\left[-\frac{\lambda}{2}+\eta_{1} \tanh \left(\eta_{1} \xi+\xi_{0}\right)\right] \\
& +\frac{2}{25} \frac{v\left(210 c \lambda^{3}+120 c \mu \lambda+12 v \lambda^{2}+45 c \lambda+12 \mu v+2 v\right)}{\lambda^{2}\left(14 \lambda^{2}+8 \mu+3\right)}\left[-\frac{\lambda}{2}+\eta_{1} \tanh \left(\eta_{1} \xi+\xi_{0}\right)\right]^{2} \\
& +\frac{4}{25} \frac{\mu v\left(210 c \lambda^{3}+120 c \mu \lambda+6 v \lambda^{2}+45 c \lambda+6 \mu v+v\right)}{\lambda\left(14 \lambda^{2}+8 \mu+3\right)}\left[-\frac{\lambda}{2}+\eta_{1} \tanh \left(\eta_{1} \xi+\xi_{0}\right)\right]^{-1} \\
& +\frac{2}{25} \frac{\mu^{2} v\left(210 c \lambda^{3}+120 c \mu \lambda+12 v \lambda^{2}+45 c \lambda+12 \mu v+2 v\right)}{\lambda^{2}\left(14 \lambda^{2}+8 \mu+3\right)}\left[-\frac{\lambda}{2}+\eta_{1} \tanh \left(\eta_{1} \xi+\xi_{0}\right)\right]^{-2},
\end{aligned}
$$


when $\left|C_{2} / C_{1}\right|<1$ and $\tanh \left(\xi_{0}\right)=C_{2} / C_{1}$;

$$
\begin{aligned}
U_{12}(\xi)= & \frac{1}{150} \frac{\left[180 v^{2} \lambda^{4}+324 \mu v^{2} \lambda^{2}+144 \mu^{2} v^{2}+54 v^{2} \lambda^{2}-1050 \lambda^{4}+48 \mu v^{2}-600 \mu \lambda^{2}+4 v^{2}-225 \lambda^{2}\right]}{\lambda^{2}\left(14 \lambda^{2}+8 \mu+3\right)} \\
& +\frac{4}{25} \frac{v^{2}\left(6 \lambda^{2}+6 \mu+1\right)}{\lambda\left(14 \lambda^{2}+8 \mu+3\right)}\left[-\frac{\lambda}{2}+\eta_{1} \operatorname{coth}\left(\eta_{1} \xi+\xi_{0}\right)\right] \\
& +\frac{2}{25} \frac{v\left(210 c \lambda^{3}+120 c \mu \lambda+12 v \lambda^{2}+45 c \lambda+12 \mu v+2 v\right)}{\lambda^{2}\left(14 \lambda^{2}+8 \mu+3\right)}\left[-\frac{\lambda}{2}+\eta_{1} \operatorname{coth}\left(\eta_{1} \xi+\xi_{0}\right)\right]^{2} \\
& +\frac{4}{25} \frac{\mu v\left(210 c \lambda^{3}+120 c \mu \lambda+6 v \lambda^{2}+45 c \lambda+6 \mu v+v\right)}{\lambda\left(14 \lambda^{2}+8 \mu+3\right)}\left[-\frac{\lambda}{2}+\eta_{1} \operatorname{coth}\left(\eta_{1} \xi+\xi_{0}\right)\right]^{-1} \\
& +\frac{2}{25} \frac{\mu^{2} v\left(210 c \lambda^{3}+120 c \mu \lambda+12 v \lambda^{2}+45 c \lambda+12 \mu v+2 v\right)}{\lambda^{2}\left(14 \lambda^{2}+8 \mu+3\right)}\left[-\frac{\lambda}{2}+\eta_{1} \operatorname{coth}\left(\eta_{1} \xi+\xi_{0}\right)\right]^{-2},
\end{aligned}
$$

when $\left|C_{2} / C_{1}\right|>1$ and $\operatorname{coth}\left(\xi_{0}\right)=C_{2} / C_{1}$.

(ii) Case 2. When $\lambda^{2}-4 \mu<0$, the solution of the trigonometric form of (13) is as follows:

$$
\begin{aligned}
U_{2}(\xi)= & \frac{1}{150} \frac{\left[180 v^{2} \lambda^{4}+324 \mu v^{2} \lambda^{2}+144 \mu^{2} v^{2}+54 v^{2} \lambda^{2}-1050 \lambda^{4}+48 \mu v^{2}-600 \mu \lambda^{2}+4 v^{2}-225 \lambda^{2}\right]}{\lambda^{2}\left(14 \lambda^{2}+8 \mu+3\right)} \\
& +\frac{4}{25} \frac{v^{2}\left(6 \lambda^{2}+6 \mu+1\right)}{\lambda\left(14 \lambda^{2}+8 \mu+3\right)}\left[-\frac{\lambda}{2}+\eta_{2}\left(\frac{-C_{1} \sin \left(\eta_{2} \xi\right)+C_{2} \cos \left(\eta_{2} \xi\right)}{C_{1} \cos \left(\eta_{2} \xi\right)+C_{2} \sin \left(\eta_{2} \xi\right)}\right)\right] \\
& +\frac{2}{25} \frac{v\left(210 c \lambda^{3}+120 c \mu \lambda+12 v \lambda^{2}+45 c \lambda+12 \mu v+2 v\right)}{\lambda^{2}\left(14 \lambda^{2}+8 \mu+3\right)}\left[-\frac{\lambda}{2}+\eta_{2}\left(\frac{-C_{1} \sin \left(\eta_{2} \xi\right)+C_{2} \cos \left(\eta_{2} \xi\right)}{C_{1} \cos \left(\eta_{2} \xi\right)+C_{2} \sin \left(\eta_{2} \xi\right)}\right)\right]^{2} \\
& +\frac{4}{25} \frac{\mu v\left(210 c \lambda^{3}+120 c \mu \lambda+6 v \lambda^{2}+45 c \lambda+6 \mu v+v\right)}{\lambda\left(14 \lambda^{2}+8 \mu+3\right)}\left[-\frac{\lambda}{2}+\eta_{2}\left(\frac{-C_{1} \sin \left(\eta_{2} \xi\right)+C_{2} \cos \left(\eta_{2} \xi\right)}{C_{1} \cos \left(\eta_{2} \xi\right)+C_{2} \sin \left(\eta_{2} \xi\right)}\right)\right]^{-1} \\
& +\frac{2}{25} \frac{\mu^{2} v\left(210 c \lambda^{3}+120 c \mu \lambda+12 v \lambda^{2}+45 c \lambda+12 \mu v+2 v\right)}{\lambda^{2}\left(14 \lambda^{2}+8 \mu+3\right)}\left[-\frac{\lambda}{2}+\eta_{2}\left(\frac{-C_{1} \sin \left(\eta_{2} \xi\right)+C_{2} \cos \left(\eta_{2} \xi\right)}{C_{1} \cos \left(\eta_{2} \xi\right)+C_{2} \sin \left(\eta_{2} \xi\right)}\right)\right]^{-2},
\end{aligned}
$$


where $\xi=x-c t, \eta_{2}=(1 / 2) \sqrt{4 \mu-\lambda^{2}}$, and $C_{1}$ and $C_{2}$ are free constants. (iii) Case 3. When $\lambda^{2}-4 \mu=0$, the solution to (13), in rational functional form, is as follows:

$$
\begin{aligned}
U_{3}(\xi)= & \frac{1}{150} \frac{\left[180 v^{2} \lambda^{4}+324 \mu v^{2} \lambda^{2}+144 \mu^{2} v^{2}+54 v^{2} \lambda^{2}-1050 \lambda^{4}+48 \mu v^{2}-600 \mu \lambda^{2}+4 v^{2}-225 \lambda^{2}\right]}{\lambda^{2}\left(14 \lambda^{2}+8 \mu+3\right)} \\
& +\frac{4}{25} \frac{v^{2}\left(6 \lambda^{2}+6 \mu+1\right)}{\lambda\left(14 \lambda^{2}+8 \mu+3\right)}\left[-\frac{\lambda}{2}+\frac{C_{2}}{C_{1}+C_{2} \xi}\right] \\
& +\frac{2}{25} \frac{v\left(210 c \lambda^{3}+120 c \mu \lambda+12 v \lambda^{2}+45 c \lambda+12 \mu v+2 v\right)}{\lambda^{2}\left(14 \lambda^{2}+8 \mu+3\right)}\left[-\frac{\lambda}{2}+\frac{C_{2}}{C_{1}+C_{2} \xi}\right]^{2} \\
& +\frac{4}{25} \frac{\mu v\left(210 c \lambda^{3}+120 c \mu \lambda+6 v \lambda^{2}+45 c \lambda+6 \mu v+v\right)}{\lambda\left(14 \lambda^{2}+8 \mu+3\right)}\left[-\frac{\lambda}{2}+\frac{C_{2}}{C_{1}+C_{2} \xi}\right]^{-1} \\
& +\frac{2}{25} \frac{\mu^{2} v\left(210 c \lambda^{3}+120 c \mu \lambda+12 v \lambda^{2}+45 c \lambda+12 \mu v+2 v\right)}{\lambda^{2}\left(14 \lambda^{2}+8 \mu+3\right)}\left[-\frac{\lambda}{2}+\frac{C_{2}}{C_{1}+C_{2} \xi}\right]^{-2},
\end{aligned}
$$

where $\xi=x-c t$ and $C_{1}, C_{2}$ are free constants.

\subsection{The IMBq Equation with Stokes Damping}

$$
u_{t t}-u_{x x}-u_{x x t t}+v u_{t}=\left(u^{3}\right)_{x x}
$$

where $v$ is a nonnegative number. We apply (7) to (25), we integrate it once concerning $\xi$, we set the constant to $M$, and then we can convert (25) to the nonlinear ODE:

$$
\left(c^{2}-1\right) U^{\prime}-c^{2} U^{\prime \prime \prime}-c v U-3 U^{2} U^{\prime}=M .
$$

At equilibrium, the highest nonlinear term $U^{2} U^{\prime}$ in (26), and the highest derivative term $U^{\prime \prime \prime}$ in (26), we get $m$ equal to 1. Therefore, the exact solution of (26) in the form of (9) can be written as follows:

$$
U(\xi)=a_{0}+a_{1} \frac{G \prime}{G}+b_{1} \frac{G}{G^{\prime}} .
$$

Substitute (27) into (26), use the ordinary differential (10) concerning $\left(G^{\prime} / G\right)$ to combine the same power terms of $\left(G^{\prime} / G\right)$, and then let the coefficients be equal to zero; we get a nonlinear algebraic system of equations concerning the unknowns $a_{0}, a_{1}, b_{1}, c$, and $M$ :

$$
\begin{aligned}
& \left(\frac{G}{G \prime}\right)^{5}:-24 c^{2} \mu^{3} b_{2}-4 \mu b_{2}^{2}=0 \\
& \left(\frac{G}{G \prime}\right)^{4}:-6 c^{2} \mu^{3} b_{1}-54 c^{2} \mu^{2} \lambda b_{2}+6 c \mu^{2} v b_{2}-6 \mu b_{1} b_{2}-4 \lambda b_{2}^{2}=0 \\
& \left(\frac{G}{G \prime}\right)^{3}:-12 c^{2} \mu^{2} \lambda b_{1}-38 c^{2} \mu \lambda^{2} b_{2}-40 c^{2} \mu^{2} b_{2}+2 c \mu^{2} v b_{1}+10 c \mu v \lambda b_{2}+2 c^{2} \mu b_{2}-4 \mu a_{0} b_{2}-2 \mu b_{1}^{2}-6 \lambda b_{1} b_{2}-2 \mu b_{2}-4 b_{2}^{2}=0 \\
& \left(\frac{G}{G \prime}\right)^{2}:-7 c^{2} \mu \lambda^{2} b_{1}-8 c^{2} \lambda^{3} b_{2}-8 c^{2} \mu^{2} b_{1}-52 c^{2} \mu \lambda b_{2}+3 c \mu v \lambda b_{1}+4 c v \lambda^{2} b_{2}+c^{2} \mu b_{1}+2 c^{2} \lambda b_{2}+8 c \mu v b_{2} \\
& \quad-2 \mu a_{0} b_{1}-2 \mu a_{1} b_{2}-4 \lambda a_{0} b_{2}-2 \lambda b_{1}^{2}-\mu b_{1}-2 \lambda b_{2}-6 b_{1} b_{2}=0
\end{aligned}
$$




$$
\begin{aligned}
& \frac{G}{G^{\prime}}:-c^{2} \lambda^{3} b_{1}-8 c^{2} \mu \lambda b_{1}-14 c^{2} \lambda^{2} b_{2}+c v \lambda^{2} b_{1}-16 c^{2} \mu b_{2}+c^{2} \lambda b_{1}+2 c \mu v b_{1}+6 c v \lambda b_{2}+2 c^{2} b_{2}-2 \lambda a_{0} b_{1}-2 \lambda a_{1} b_{2} \\
&-\lambda b_{1}-4 a_{0} b_{2}-2 b_{1}^{2}-2 b_{2}=0, \\
&\left(\frac{G}{G^{\prime}}\right)^{0}: b_{1} c^{2}-2 a_{0} b_{1}+2 a_{2} b_{1} \mu+c v a_{1} \lambda \mu-c^{2} b_{1} \lambda^{2}-2 c^{2} \mu b_{1}-6 c^{2} \lambda b_{2}+2 c^{2} a_{1} \mu^{2}-c^{2} \mu a_{1}+2 a_{0} a_{1} \mu+2 c v b_{2} \\
&+\mu a_{1}+c^{2} a_{1} \lambda^{2} \mu+6 c^{2} a_{2} \lambda \mu^{2}-2 a_{1} b_{2}+c v b_{1} \lambda+2 c v a_{2} \mu^{2}-b_{1}-M=0, \\
& \frac{G^{\prime}}{G}: 14 c^{2} \mu \lambda^{2} a_{2}+c^{2} \lambda^{3} a_{1}+16 c^{2} \mu^{2} a_{2}+8 c^{2} \mu \lambda a_{1}+6 c \mu v \lambda a_{2}+c v \lambda^{2} a_{1}-2 c^{2} \mu a_{2}-c^{2} \lambda a_{1}+2 c \mu v a_{1} \\
&+4 \mu a_{0} a_{2}+2 \mu a_{1}^{2}+2 \lambda a_{0} a_{1}+2 \lambda a_{2} b_{1}+2 \mu a_{2}+\lambda a_{1}=0, \\
&\left(\frac{G^{\prime}}{G}\right)^{2}: 8 c^{2} \lambda^{3} a_{2}+52 c^{2} \mu \lambda a_{2}+7 c^{2} \lambda^{2} a_{1}+4 c v \lambda^{2} a_{2}+8 c^{2} \mu a_{1}-2 c^{2} \lambda a_{2}+8 c \mu v a_{2}+3 c v \lambda a_{1}-c^{2} a_{1}+6 \mu a_{1} a_{2} \\
&+4 \lambda a_{0} a_{2}+2 \lambda a_{1}^{2}+2 \lambda a_{2}+2 a_{0} a_{1}+2 a_{2} b_{1}+a_{1}=0, \\
&\left(\frac{G^{\prime}}{G}\right)^{3}: 38 c^{2} \lambda^{2} a_{2}+40 c^{2} \mu a_{2}+12 c^{2} \lambda a_{1}+10 c v \lambda a_{2}-2 c^{2} a_{2}+2 c v a_{1}+4 \mu a_{2}^{2}+6 \lambda a_{1} a_{2}+4 a_{0} a_{2}+2 a_{1}^{2}+2 a_{2}=0, \\
&\left(\frac{G^{\prime}}{G}\right)^{4}: 54 c^{2} \lambda a_{2}+6 c^{2} a_{1}+6 c v a_{2}+4 \lambda a_{2}^{2}+6 a_{1} a_{2}=0, \\
&\left(\frac{G^{\prime}}{G}\right)^{5}: 24 c^{2} a_{2}+4 a_{2}^{2}=0 .
\end{aligned}
$$

Using symbolic computation software maple, the solution of this system is obtained as follows:

Case 1: $\left\{a_{0}=-I, a_{1}=0, b_{1}=-\frac{-I c v+M}{c^{2} \lambda^{2}+2 c^{2} \mu-c^{2}-2}\right\}$,

Case 2: $\left\{a_{0}=I, a_{1}=0, b_{1}=-\frac{I c v+M}{c^{2} \lambda^{2}+2 c^{2} \mu-c^{2}-2}\right\}$, where $I$ is the imaginary unit. Substituting the values from (29) and using the general solutions of (10) in different situations, multiple exact solutions of different types of (25) can be obtained.

(i) Case 1. When $\lambda^{2}-4 \mu>0$, the solution to (25), in hyperbolic functional form, is as follows:

$$
U_{1}(\xi)=-I-\frac{-I c v+M}{c^{2} \lambda^{2}+2 c^{2} \mu-c^{2}-2}\left[-\frac{\lambda}{2}+\eta_{1}\left(\frac{C_{1} \sinh \left(\eta_{1} \xi\right)+C_{2} \cosh \left(\eta_{1} \xi\right)}{C_{1} \cosh \left(\eta_{1} \xi\right)+C_{2} \sinh \left(\eta_{1} \xi\right)}\right)\right]^{-1}
$$

where $\xi=x-c t, \eta_{1}=(1 / 2) \sqrt{\lambda^{2}-4 \mu}$, and $C_{1}, C_{2}$ are free constants.

In particular, if $C_{1} \neq 0$ and $C_{2}=0$, then $U_{1}(\xi)$ becomes

$$
\bar{U}_{1}(\xi)=-I-\frac{-I c v+M}{c^{2} \lambda^{2}+2 c^{2} \mu-c^{2}-2}\left[-\frac{\lambda}{2}+\eta_{1} \tanh \left(\eta_{1} \xi\right)\right]^{-1} \text {. }
$$

Again using (12), the general solutions for $U_{1}(\xi)$ in simplified forms are written as

$$
U_{11}(\xi)=-I-\frac{-I c v+M}{c^{2} \lambda^{2}+2 c^{2} \mu-c^{2}-2}\left[-\frac{\lambda}{2}+\eta_{1} \tanh \left(\eta_{1} \xi+\xi_{0}\right)\right]^{-1},
$$

when $\left|C_{2} / C_{1}\right|<1$ and $\tanh \left(\xi_{0}\right)=C_{2} / C_{1}$;

$$
U_{12}(\xi)=-I-\frac{-I c v+M}{c^{2} \lambda^{2}+2 c^{2} \mu-c^{2}-2}\left[-\frac{\lambda}{2}+\eta_{1} \operatorname{coth}\left(\eta_{1} \xi+\xi_{0}\right)\right]^{-1},
$$

when $\left|C_{2} / C_{1}\right|>1$ and $\operatorname{coth}\left(\xi_{0}\right)=C_{2} / C_{1}$. 
(ii) Case 2. When $\lambda^{2}-4 \mu<0$, the solution to (25), in trigonometric functional form, is as follows:

$$
U_{2}(\xi)=-I-\frac{-I c v+M}{c^{2} \lambda^{2}+2 c^{2} \mu-c^{2}-2}\left[-\frac{\lambda}{2}+\eta_{2}\left(\frac{-C_{1} \sin \left(\eta_{2} \xi\right)+C_{2} \cos \left(\eta_{2} \xi\right)}{C_{1} \cos \left(\eta_{2} \xi\right)+C_{2} \sin \left(\eta_{2} \xi\right)}\right)\right]^{-1},
$$

where $\xi=x-c t, \eta_{2}=(1 / 2) \sqrt{4 \mu-\lambda^{2}}$, and $C_{1}, C_{2}$ are free constants.

(iii) Case 3. When $\lambda^{2}-4 \mu=0$, the solution to (25), in rational functional form, is as follows:

$$
U_{3}(\xi)=-I-\frac{-I c v+M}{c^{2} \lambda^{2}+2 c^{2} \mu-c^{2}-2}\left[-\frac{\lambda}{2}+\frac{C_{2}}{C_{1}+C_{2} \xi}\right]^{-1}
$$

where $\xi=x-c t$ and $C_{1}, C_{2}$ are arbitrary constants.

Substituting the values from (30) and using the general solutions of (10) in different situations, multiple exact solutions of different types of (25) can be obtained.

(i) Case 1. When $\lambda^{2}-4 \mu>0$, the solution to (25), in hyperbolic functional form, is as follows:

$$
U_{1}(\xi)=I-\frac{I c v+M}{c^{2} \lambda^{2}+2 c^{2} \mu-c^{2}-2}\left[-\frac{\lambda}{2}+\eta_{1}\left(\frac{C_{1} \sinh \left(\eta_{1} \xi\right)+C_{2} \cosh \left(\eta_{1} \xi\right)}{C_{1} \cosh \left(\eta_{1} \xi\right)+C_{2} \sinh \left(\eta_{1} \xi\right)}\right)\right]^{-1}
$$

where $\xi=x-c t, \eta_{1}=(1 / 2) \sqrt{\lambda^{2}-4 \mu}$, and $C_{1}, C_{2}$ are free constants.

In particular, if $C_{1} \neq 0$ and $C_{2}=0$, then $U_{1}(\xi)$ becomes

$$
\bar{U}_{1}(\xi)=I-\frac{I c v+M}{c^{2} \lambda^{2}+2 c^{2} \mu-c^{2}-2}\left[-\frac{\lambda}{2}+\eta_{1} \tanh \left(\eta_{1} \xi\right)\right]^{-1} \text {. }
$$

Again using (12), the general solutions for $U_{1}(\xi)$ in simplified forms are written as

$$
U_{11}(\xi)=I-\frac{I c v+M}{c^{2} \lambda^{2}+2 c^{2} \mu-c^{2}-2}\left[-\frac{\lambda}{2}+\eta_{1} \tanh \left(\eta_{1} \xi+\xi_{0}\right)\right]^{-1},
$$

when $\left|C_{2} / C_{1}\right|<1$ and $\tanh \left(\xi_{0}\right)=C_{2} / C_{1}$;

$$
U_{12}(\xi)=I-\frac{I c v+M}{c^{2} \lambda^{2}+2 c^{2} \mu-c^{2}-2}\left[-\frac{\lambda}{2}+\eta_{1} \operatorname{coth}\left(\eta_{1} \xi+\xi_{0}\right)\right]^{-1},
$$

when $\left|C_{2} / C_{1}\right|>1$ and $\operatorname{coth}\left(\xi_{0}\right)=C_{2} / C_{1}$.

(ii) Case 2. When $\lambda^{2}-4 \mu<0$, the solution to (25), in trigonometric functional form, is as follows:

$$
U_{2}(\xi)=I-\frac{I c v+M}{c^{2} \lambda^{2}+2 c^{2} \mu-c^{2}-2}\left[-\frac{\lambda}{2}+\eta_{2}\left(\frac{-C_{1} \sin \left(\eta_{2} \xi\right)+C_{2} \cos \left(\eta_{2} \xi\right)}{C_{1} \cos \left(\eta_{2} \xi\right)+C_{2} \sin \left(\eta_{2} \xi\right)}\right)\right]^{-1}
$$

where $\xi=x-c t, \eta_{2}=(1 / 2) \sqrt{4 \mu-\lambda^{2}}$, and $C_{1}, C_{2}$ are arbitrary constants.

(iii) Case 3. When $\lambda^{2}-4 \mu=0$, the solution to (25), in rational functional form, is as follows:

$$
U_{3}(\xi)=I-\frac{I c v+M}{c^{2} \lambda^{2}+2 c^{2} \mu-c^{2}-2}\left[-\frac{\lambda}{2}+\frac{C_{2}}{C_{1}+C_{2} \xi}\right]^{-1},
$$

where $\xi=x-c t$ and $C_{1}, C_{2}$ are free constants.

\section{Discussion}

In [14], the damped IBq (14) is derived and the local waveform of its numerical solution is obtained. The local two-dimensional display of the numerical solution is a bellshaped waveform, similar to the local figure of the twodimensional figure obtained by taking time as a constant in Figure 1. Figure 1 is a three-dimensional diagram of the trigonometric solution (23). It is well known that numerical solutions may miss some solutions of the equation, as shown in Figure 2, which is a kinked type of isolated wave and is not mentioned in [14]. As can be seen from Figure 2, when we 


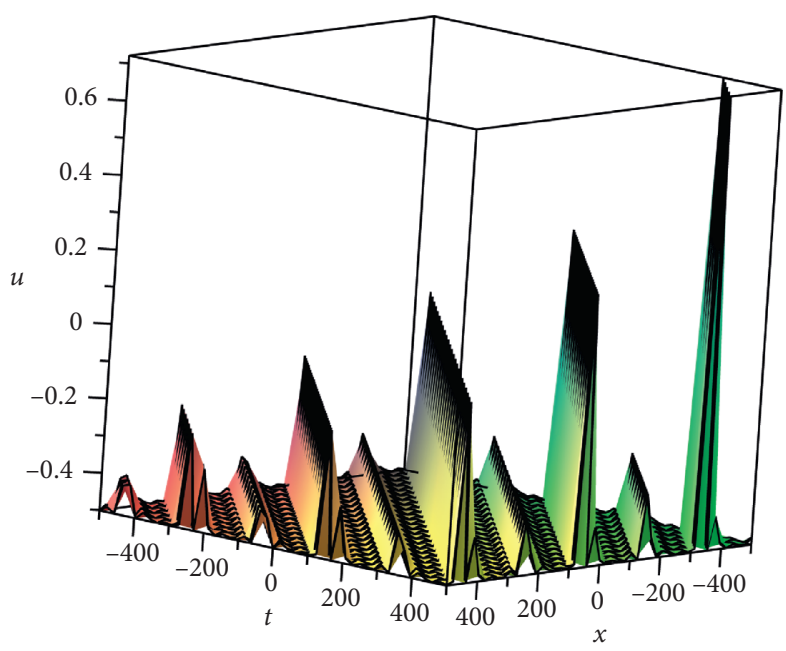

Figure 1: 3D graph of solution (23) when $\lambda=\sqrt{2}, \mu=1.5, c=1, v=0.001$, and $C_{1}=C_{2}=1$.

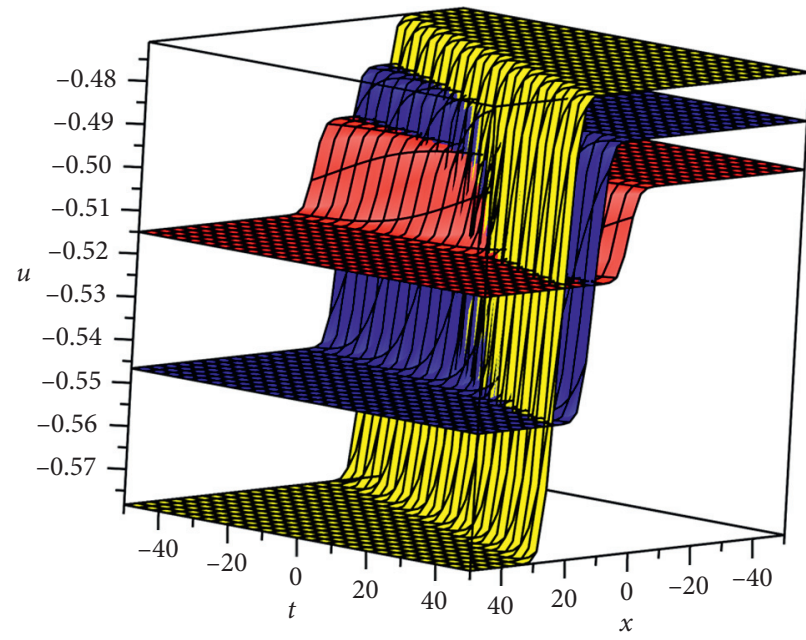

Solution (18) red: $c=0.1$, blue: $c=0.3$, and yellow: $c=0.5$

Figure 2: 3D graph of solution (19) when $\lambda=\sqrt{2}, \mu=0.3, v=0.1$, and $C_{2}=0$.

change the value of wave velocity $c$ and increase gradually, the propagation direction of kinked isolated wave follows the change, and the amplitude of waveform increases gradually. Mathematically, the value of $c$ can be used to modulate the kinked isolated waveform represented by this equation.

In [15], the authors deduce a damped equation similar to (14) and use a numerical method (finite difference method) to find a kinked isolated wave. The equation derived in [15] is (43), and it is also concluded that when the dissipation coefficient $\beta_{1}$ is much larger than the dispersion coefficient $\beta_{2}$, the kinked isolated wave will have asymmetric characteristics:

$$
u_{t t}-u_{x x}+u_{x x x x}-\beta_{2} u_{x x t t}-\beta_{1} u_{x x t}=\left(u^{2}\right)_{x x} .
$$

Using (7), we apply the travelling wave transformation to (13) and (43), integrate once, and then assume that $\beta_{1}=v$ and $\beta_{2}=1+c^{-2}$; we get the same (14). The waveform of the kinked isolated wave obtained from (14) varies with the damping coefficient $v$, as shown in Figure 3. As can be seen from Figure 3, with the increase of $v$, the precise kink solitary wave solution waveform obtained by the extended $\left(G^{\prime} / G\right)$ expansion also shows the asymmetric characteristics of waveform and becomes more and more obvious. It is shown that the exact solution is helpful to verify the numerical solution.

It can be seen from solution (17) of the algebraic system of (14) that $a_{0}, a_{1}, a_{2}, b_{1}$, and $b_{2}$ are expressed by other parameters. When we want to find an expression for wave velocity $c$, we can also find a solution in the following form. The difference is that, in the expression for solution (17), the wave velocity is arbitrary, and here the coefficient $a_{2}$ is arbitrary: 


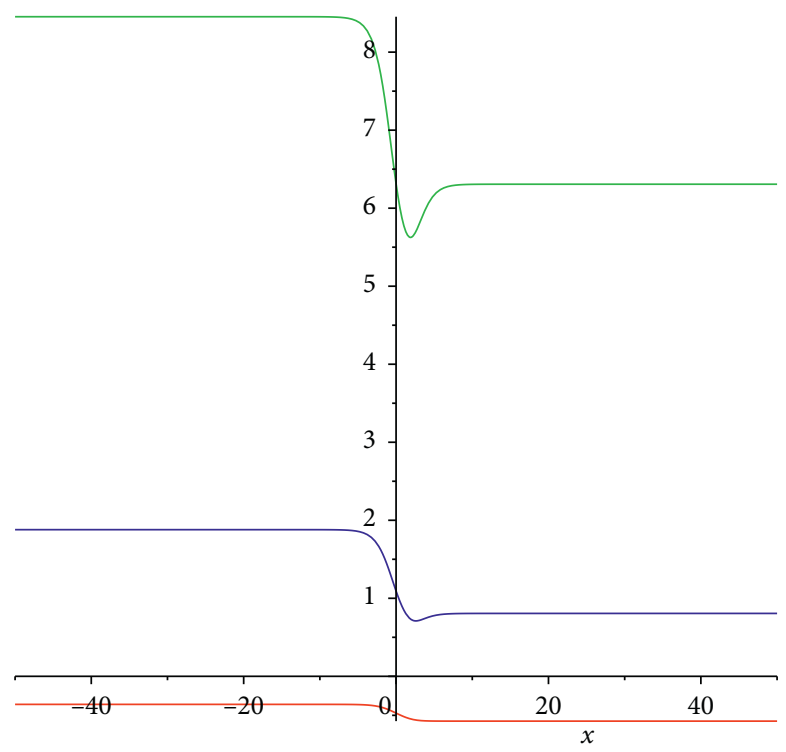

Solution (18) red: $v=1$, blue: $v=5$, and green: $v=10$

Figure 3: The two-dimensional representation of solution (19) when $\lambda=\sqrt{2}, \mu=0.3, c=0.1, t=10$, and $C_{2}=0$.

$$
\left\{\begin{array}{c}
c=-\frac{1}{30} \frac{-350 \lambda^{4} a_{2}-200 \mu \lambda^{2} a_{2}+24 v^{2} \lambda^{2}+24 \mu v^{2}-75 \lambda^{2} a_{2}+4 v^{2}}{\left(14 \lambda^{2}+8 \mu+3\right) v \lambda}, \\
a_{0}=\frac{1}{150} \frac{\left[180 v^{2} \lambda^{4}+324 \mu v^{2} \lambda^{2}+144 \mu^{2} v^{2}+54 v^{2} \lambda^{2}-1050 \lambda^{4}+48 \mu v^{2}-600 \mu \lambda^{2}+4 v^{2}-225 \lambda^{2}\right]}{\lambda^{2}\left(14 \lambda^{2}+8 \mu+3\right)}, \\
a_{1}=\frac{4}{25} \frac{v^{2}\left(6 \lambda^{2}+6 \mu+1\right)}{\lambda\left(14 \lambda^{2}+8 \mu+3\right)}, \\
b_{1}=-\frac{2}{25} \frac{\left(-350 \lambda^{4} a_{2}-200 \mu \lambda^{2} a_{2}+12 v^{2} \lambda^{2}+12 \mu v^{2}-75 \lambda^{2} a_{2}+2 v^{2}\right) \mu}{\left(14 \lambda^{2}+8 \mu+3\right) \lambda} .
\end{array}\right\}
$$

When we use solution (44), the arbitrary constant $M$ also contains an expression for $a_{2}$, which is too long to display here. For solutions (29) and (30) of the second algebraic system, the expressions are simple, and the expressions of arbitrary constants $M$ and wave velocity $c$ can be easily solved from them.

In the extended $\left(G^{\prime} / G\right)$-expansion method, when the coefficients $b_{i}(i=1,2, \ldots, m)$ are set to 0 , it is reduced to the $\left(G^{\prime} / G\right)$-expansion method, which is the same idea as the simplest equation method reduced to the tanh method [43]. We think superficially that the extended $\left(G^{\prime} / G\right)$-expansion method fuses the solutions of two Ricatti equations in a certain form. In other words, the extended $\left(G^{\prime} / G\right)$-expansion method decomposes the solutions of nonlinear partial differential equation into the solutions of two Ricatti equations. A brief explanation is given as follows.

If $G=G(\xi)$ is the solution of the second-order differential equation

$$
G^{\prime \prime}+\lambda G^{\prime}+\mu G=0
$$

we can get 


$$
\begin{aligned}
\left(\frac{G^{\prime}(\xi)}{G(\xi)}\right)^{\prime} & =\frac{G^{\prime \prime}(\xi) G(\xi)-\left(G^{\prime}(\xi)\right)^{2}}{G^{2}(\xi)}=-\left(\frac{G^{\prime}}{G}\right)^{2}-\lambda\left(\frac{G^{\prime}}{G}\right)-\mu \\
\left(\frac{G(\xi)}{G^{\prime}(\xi)}\right)^{\prime} & =\frac{\left(G^{\prime}(\xi)\right)^{2}-G(\xi) G^{\prime \prime}(\xi)}{\left(G^{\prime}(\xi)\right)^{2}}=1+\frac{G\left(\lambda G^{\prime}+\mu G\right)}{\left(G^{\prime}\right)^{2}} \\
& =1+\lambda\left(\frac{G}{G^{\prime}}\right)+\mu\left(\frac{G}{G^{\prime}}\right)^{2}
\end{aligned}
$$

It can be seen from (46) and (47) that when $G$ satisfies (45), $\left(G^{\prime} / G\right)$ and $\left(G / G^{\prime}\right)$ each satisfy a generalized Ricatti equation, and the derivative results of $\left(G^{\prime} / G\right)$ and $\left(G / G^{\prime}\right)$ can be converted into the polynomial form of $\left(G^{\prime} / G\right)$ or $\left(G / G^{\prime}\right)$, which is the basis for collecting coefficients to obtain an algebraic system of equations.

\section{Conclusion}

With the help of the auxiliary function method, we solved two damped generalized IBq equations and we obtained their three types of travelling wave exact solutions, which is an extension work of the numerical solution and the existence of a solution. From the waveform of solution (19), it can be seen that the waveform of the kink wave can be modulated by changing the value of $c$. It is found that, for the hydrodynamic damping IBq equation, the varying damping coefficient $v$ makes the waveform of the kinked isolated wave represented by (19) appear obviously asymmetric, which is consistent with the conclusion obtained by numerical method in [15]. In addition, the role of maple in our work should not be overlooked to make our computing work easier.

\section{Data Availability}

No data were used to support this study.

\section{Conflicts of Interest}

The authors declare that they have no conflicts of interest.

\section{Authors' Contributions}

All authors read and approved the final manuscript.

\section{Acknowledgments}

This research was supported by Major Science and Technology Projects in Shanxi Province of China (20181101008 and 20181102015) and Supplementary Platform Project of "1331" Project in Shanxi Province in 2018.

\section{References}

[1] J. Boussinesq, "Théorie des ondes et des remous qui se propagent le long d'un canal rectangulaire horizontal en communiquant au liquide contenu dans ce canal des vitesses sensiblement pareilles de la surface au fond," Journal de
Mathématiques Pures et Appliquées, vol. 17, no. 2, pp. 55-108, 1872.

[2] J. Boussinesq, "Essai sur la théorie des eaux courantes," Mém. Acad. Sci. Inst. Nat. France, vol. 23, no. 1, pp. 1-680, 1877.

[3] V. Varlamov, "Eigenfunction expansion method and the longtime asymptotics for the damped Boussinesq equation," Discrete \& Continuous Dynamical Systems-A, vol. 7, no. 4, pp. 675-702, 2001.

[4] T. Hatice, N. Polat, and A. Ertaş, "Global existence and decay of solutions for the generalized bad Boussinesq equation," Applied Mathematics-A Journal of Chinese Universities, vol. 28, no. 3, pp. 253-268, 2013.

[5] A.-M. Wazwaz, "Compactons and solitary wave solutions for the Boussinesq wave equation and its generalized form," Applied Mathematics and Computation, vol. 182, no. 1, pp. 529-535, 2006.

[6] S. T. Mohyud, M. A. Noor, and A. Waheed, "Exp-function method for generalized traveling solutions of good Boussinesq equations," Journal of Applied Mathematics and Computing, vol. 30, no. 1-2, pp. 439-445, 2009.

[7] G. Forozani and M. Ghorveei Nosrat, "Solitary solution of modified bad and good Boussinesq equation by using of tanh and extended tanh methods," Indian Journal of Pure and Applied Mathematics, vol. 44, no. 4, pp. 497-510, 2013.

[8] V. G. Makhankov, "Dynamics of classical solitons (in nonintegrable systems)," Physics Reports, vol. 35, no. 1, pp. 1-128, 1978.

[9] P. Chatterjee, R. Ali, and A. Saha, "Analytical solitary wave solution of the dust ion acoustic waves for the damped forced korteweg-de Vries equation in superthermal plasmas," Zeitschrift für Naturforschung A, vol. 73, no. 2, pp. 151-159, 2018.

[10] R. Ali, A. Saha, and P. Chatterjee, "Analytical electron acoustic solitary wave solution for the forced $\mathrm{KdV}$ equation in superthermal plasmas," Physics of Plasmas, vol. 24, no. 12, Article ID 122106, 2017.

[11] B. Yan, P. Prasad, S. Mukherjee, A. Saha, and S. Banerjee, "Dynamical complexity and multistability in a novel lunar wake plasma system," Complexity, vol. 2020, Article ID 5428548, 2020.

[12] A. Saha and J. Tamang, "Effect of q-nonextensive hot electrons on bifurcations of nonlinear and supernonlinear ion-acoustic periodic waves," Advances in Space Research, vol. 63, no. 5, pp. 1596-1606, 2019.

[13] P. Rosenau, "Dynamics of nonlinear mass-spring chains near the continuum limit," Physics Letters A, vol. 118, no. 5, pp. 222-227, 1986.

[14] E. Arévalo, Y. Gaididei, and F. G. Mertens, "Soliton dynamics in damped and forced Boussinesq equations," The European Physical Journal B, vol. 27, no. 1, pp. 63-74, 2002.

[15] S. S. Naranmandula, "The evolution of bell and Kink isolated waves in microstructured solids," Chinese Journal of Theoretical and Applied Mechanics, vol. 44, no. 1, pp. 117-122, 2012.

[16] S. Wang and H. Xu, "On the asymptotic behavior of solution for the generalized IBq equation with hydrodynamical damped term," Journal of Differential Equations, vol. 252, no. 7, pp. 4243-4258, 2012.

[17] S. Wang and $\mathrm{H}$. Xu, "On the asymptotic behavior of solution for the generalized IBq equation with Stokes damped term," Zeitschrift für angewandte Mathematik und Physik, vol. 64, no. 3, pp. 719-731, 2013. 
[18] X. Y. Chen, "Global existence of solution of cauchy problem for a generalized IMBq equation with weak damping," Acta Mathematica Scientia, vol. 35, no. 1, pp. 68-82, 2015.

[19] M. A. E. Abdelrahman and O. Moaaz, "New exact solutions to the dual-core optical fibers," Indian Journal of Physics, vol. 94, no. 5, pp. 705-711, 2020.

[20] J. Manafian and M. Lakestani, "Optical solitons with BiswasMilovic equation for Kerr law nonlinearity," European Physical Journal Plus, vol. 130, no. 4, pp. 1-12, 2015.

[21] M. Shakeel, M. A. Iqbaol, Q. Din, Q. M. Hassan, and K. Ayub, "New exact solutions for coupled nonlinear system of ion sound and Langmuir waves," Indian Journal of Physics, vol. 96, no. 5, pp. 885-894, 2020.

[22] M. Jahani and J. Manafian, "Improvement of the Exp-function method for solving the BBM equation with time-dependent coefficients," European Physical Journal Plus, vol. 131, no. 3, pp. 1-12, 2016.

[23] M. G. Hafez, M. N. Alam, and M. A. Akbar, "Traveling wave solutions for some important coupled nonlinear physical models via the coupled Higgs equation and the Maccari system," Journal of King Saud University-Science, vol. 27, no. 2, pp. 105-112, 2015.

[24] N. H. Abdel-All, M. A.-A. Abdel-Razek, and A.-A. K. Seddeek, "Expanding the tanh-function method for solving nonlinear equations," Applied Mathematics, vol. 2, no. 9, pp. 1096-1104, 2011.

[25] M. A. Akbar, N. H. M. Ali, and S. T. Mohyud-Din, "Assessment of the further improved $\left(G^{\prime} / G\right)$-expansion method and the extended tanh-method in probing exact solutions of nonlinear PDEs," Springer Plus, vol. 2, no. 1, pp. 2-9, 2013.

[26] Ö. F. Gözükızıl and Ş. Akçağıl, "The tanh-coth method for some nonlinear pseudoparabolic equations with exact solutions," Advances in Difference Equations, vol. 143, no. 1, pp. 2-18, 2013.

[27] A. S. Abdel, E. S. Osman, and M. Khalfallah, "The homogeneous balance method and its application to the BenjaminBona-Mah-oney(BBM) equation," Applied Mathematics and Computation, vol. 217, no. 4, pp. 1385-1390, 2010.

[28] M. Jalil, F. A. Mehdi, M. Khalilian, and R. S. Jeddi, “Application of the generalized $\left(G^{\prime} / G\right)$-expansion method for nonlinear PDEs to obtaining soliton wave solution," Optik-International Journal for Light and Electron Optics, vol. 135, pp. 395-406, 2017.

[29] Z. Zhang, J. Huang, J. Zhong et al., “The extended $\left(G^{\prime} / G\right)$ expansion method and travelling wave solutions for the perturbed nonlinear Schrödinger's equation with Kerr law nonlinearity," Pramana, vol. 82, no. 6, pp. 1011-1029, 2014.

[30] J. Pang, C.-q. Bian, and L. Chao, "A new auxiliary equation method for finding travelling wave solutions to KdV equation," Applied Mathematics and Mechanics, vol. 31, no. 7, pp. 929-936, 2010.

[31] M. Mirzazadeh and M. Eslami, "Exact multisoliton solutions of nonlinear Klein-Gordon equation in $1+2$ dimensions," European Physical Journal Plus, vol. 128, no. 11, pp. 1-92, 2013.

[32] N. Taghizadeh and M. Mirzazadeh, "The first integral method to some complex nonlinear partial differential equations," Journal of Computational and Applied Mathematics, vol. 235, no. 16, pp. 4871-4877, 2011.

[33] N. A. Muhammad, M. H. Syed, A. Saha et al., "Exact solutions, conservation laws, bifurcation of nonlinear and supernonlinear traveling waves for Sharma-Tasso-Olver equation," Nonlinear Dynamics, vol. 94, no. 3, pp. 1791-1801, 2018.

[34] M. Mirzazadeh and M. E. Dark, "Optical solitons of Biswas-Milovic equation with dual-power law nonlinearity,"
European Physical Journal Plus, vol. 130, no. 1, pp. 1-7, Article ID 4, 2015.

[35] M. E. Elsayed and A. N. Abdul-Ghani, "Solitons and other exact solutions for variant nonlinear Boussinesq equations," Optik-International Journal for Light and Electron Optics, vol. 139, pp. 166-177, 2017.

[36] A. Malik, F. Chand, H. Kumar, and S. C. Mishra, "Exact solutions of some physical models using the $\left(G^{\prime} / G\right)$-expansion method," Pramana, vol. 78, no. 4, pp. 513-529, 2012.

[37] D. M. Mothibi and C. M. Khalique, "On the exact solutions of a modified Kortweg de Vries type equation and higher-order modified Boussinesq equation with damping term," Advances in Difference Equations, vol. 166, no. 1, pp. 1-7, 2013.

[38] M. Wang, X. Li, and J. Zhang, “The $\left(G^{\prime} / G\right)$-expansion method and travelling wave solutions of nonlinear evolution equations in mathematical physics," Physics Letters A, vol. 372, no. 4, pp. 417-423, 2008.

[39] N. A. Kudryashov, "Seven common errors in finding exact solutions of nonlinear differential equations," Communications in Nonlinear Science and Numerical Simulation, vol. 14, no. 9-10, pp. 3507-3529, 2009.

[40] N. A. Kudryashov, "On "new travelling wave solutions" of the $\mathrm{KdV}$ and the KdV-burgers equations," Communications in Nonlinear Science and Numerical Simulation, vol. 14, no. 5, pp. 1891-1900, 2009.

[41] N. A. Kudryashov, "A note on the $G^{\prime} / G$-expansion method," Applied Mathematics and Computation, vol. 217, no. 4, pp. 1755-1758, 2010.

[42] N. A. Kudryashov and N. B. Loguinova, "Be careful with the Exp-function method," Communications in Nonlinear Science and Numerical Simulation, vol. 14, no. 5, pp. 1881-1890, 2009.

[43] N. A. Kudrayshov, "Simplest equation method to look for exact solutions of nonlinear differential equations," Chaos, Solitons \& Fractals, vol. 24, no. 5, pp. 1217-1231, 2005. 